\title{
Flame Atomic Absorption Spectrometry Based on Self-absorption in the Flame and Using the Flame as a Light Emission Source
}

\author{
Anastas Dimitrov Dakas hev ${ }^{1, *}$, Stancho Valkanov Pavlov², Krasimira Angelova Stancheva1 \\ ${ }^{1}$ Department of Inorganic and Analy tical Chemistry, Prof. Dr. Assen ZlatarovUniversity, 8010 Bourgas, Bulgaria \\ ${ }^{2}$ Department of Mathematics and Physics, Prof. Dr. Assen Zlatarov University, 8010 Bourgas, Bulgaria
}

\begin{abstract}
A method of flame atomic absorption analysis has been developed which does not need a light source, such as hollow-cathode lamp, which to produce the radiation absorbed by the analyte atoms. Light emission from the analyzed atoms in the flame can serve as a light source because this emission has the same wave length as the resonance absorption line of the unexcited analyte atoms. At certain conditions, in the flame can take place an absorption process known as self-absorption. In the work self-absorption occurring in the flame is used to determine absorbance. Absorbance is calculated from the flame emission intensity signal of the analyte atoms. A computer program is specially created to calculate absorbance, to draw the calibration curve and to compute the analyte concentration. For safety purposes ethyl alcohol is used as a flame fuel and is applied as an alcohol vapor / air flame. Because this flame has low temperature, only lithium, potassium, sodium, calcium, barium is able to be analyzed.
\end{abstract}

Keywords Flame Atomic Absorption Analysis Base on Self-absorption, Flame As a Light Emission Source for Atomic Absorption, Determination of Absorbance by Flame Emission Data

\section{Introduction}

In atomic absorption spectrometry the hollow-cathode lamp is a spectral line light source commonly used to produce the radiation absorbed by the analyte atoms. When the element to be analyzed is a non-metal, or when a hollow-cathode lamp produces a weak signal, an electrode-less discharge lamp is employed. A recently developed hollow-cathode lamp with boosted-discharge has improved analytical characteristics such as greater intensity, calibration graphs with less curvature, increased sensitivity in comparison to a conventional hollow- cathode lamp[1]. Also, a diode laser can be used as the light source for atomic absorption analysis[2]. Another current approach to performing atomic absorption analysis involves use of a continuum light source like a xenon arc lamp combined with a high resolution monochromator[3]. In the early development of atomic absorption spectroscopy, flame emission was used as a light source. This light source was prepared by aspirating a solution, containing a high concentration of the analyte, in a second flame. This method was

* Corresponding author:

adakashev@btu.bg (Anastas Dimitrov Dakashev)

Published online at http://journal.sapub.org/aac

Copyright (C) 2012 Scientific \& Academic Publishing. All Rights Reserved later applied by Clifton Calloway Jr and Brad ley Jones[4]. They claimed the light source was inexpensive and that setup changes to accommodate different analytes were quick and simple.

In the present work, one flame both atomizes the analyte and provides the light emission source.

\section{Principle of the Method}

In the flame, analyte atoms are excited and emit light. According to Kirchoff's law, the emitted light has the wave length of the resonance absorption line of unexcited atoms. As emitted light passes from some part in the interior of the flame to the outside, it is absorbed by unexcited analyte atoms. This is well known as a self-absorption process. In the present work self-absorption is used to determine absorbance. In the particular case where light emitted by the exited analyte atoms in the flame serves as a light source, absorbance $A$ is defined by the equation: $A=\log \left(I_{a t} / I_{f}\right)$, where $I_{a t}$ is the light emission intensity of the exited atoms in the flame, and $I_{f}$ is the initial emission intensity reduced by self-absorption, or emission intensity of the light that leaves the flame. The value of $I_{f}$ is measured in the conventional way, while $I_{a t}$ value has to be found in order for the absorbance to be determined. In the present work $I_{a t}$ is calculated. A computer program is specifically designed to 
make necessary calculations, as well as to draw the calibration curve, and determine the analyte concentration[5].

\section{Experimental}

\subsection{Apparatus}

An atomic absorption spectrometer Unicam SP 90 Series 2, designed also for emission measurements, is used when acetylene / air flame is applied. When ethyl alcohol is used as a flame fuel, the nebulizer and the mixing chamber of the spectrometer are replaced by a nebulizer and a mixing chamber, taken from an AAS-1N spectrometer, and adapted to the Unicam spectrometer. Using a vaporizer, the fuel is introduced in the spectrometer as an alcohol vapor. A variety of burner heads are used as follows: 1. A Meker burner head for propane / air gas mixture, mentioned in the paper as MBH 120.7, consisting of 91 openings, each of $1.2 \mathrm{~mm}$ in diameter, situated in a circle, $25 \mathrm{~mm}$ in diameter, with a total opening section area of $120.7 \mathrm{~mm}^{2}$. 2. A Meker burner head for acetylene / air gas mixture, MBH 45.5, with 58 openings, each of $1.0 \mathrm{~mm}$ in diameter, situated in a circle, $15 \mathrm{~mm}$ in diameter, with a total opening section area of 45.5 $\mathrm{mm}^{2}$. 3. A Meker burner head for acetylene / air, MBH 14.7, with 13 openings, each of $1.2 \mathrm{~mm}$ in diameter, situated in a square, $8 \times 8 \mathrm{~mm}$, with a total opening section area of 14.7 $\mathrm{mm}^{2} .4$. An acetylene / air three-slot burner head 3SBH 120, with dimensions of each slot: $50 \times 0.8 \mathrm{~mm}$, with a total opening section area of $120 \mathrm{~mm}^{2}$. 5. An acetylene / air single slot burner head, slot dimensions: $100 \times 0.8 \mathrm{~mm}$. Use is also made of a burner, named 2MBH 120.7, composed of two Meker burner heads, MBH 120.7, arranged near each other in a line coinciding with the optical line of the spectrometer. This burner gives a flame with great dimensions.

\subsection{Reagents}

All of the reagents used in the work are of analytical grade. Denatured alcohol is applied as a flame fuel.

\subsection{Solutions}

Solutions are made up in double distilled water. The stock standard solutions are prepared directly by weighing the recommended solid reagents, dissolving and diluting to the corresponding volumes with double distilled water. The working standard solutions are prepared by suitable dilution of the stock solutions with double distilled water.

\section{Results and Discussion}

The only difference between the flame atomic absorption process, occurring in ordinary flame atomic absorption analysis and the self-absorption process by the method under consideration, is the location of the light emission source. For the adsorption process, it is outside the flame but for self-adsorption process is inside it. Therefore, self-absorption must obey to the same laws as absorption.
When self-adsorption in the flame occurs, intensity of the light that leaves the flame $I_{f}$ is a nonlinear function of concentration $C$, according to Beer's law: $I_{f}=I_{a t} 10^{-a C}$, where $a$ is absorptivity. When self-absorption in the flame does not take place, flame light emission intensity $I_{f}$ is a linear function of analyte concentration (the basis of Flame emission spectrometry). That means the appearance of $I_{f}$ function is a criterion for evaluating the self-adsorption process. Experimental data of flame emission intensity for different analyte concentrations are given in Fig. 1 A.

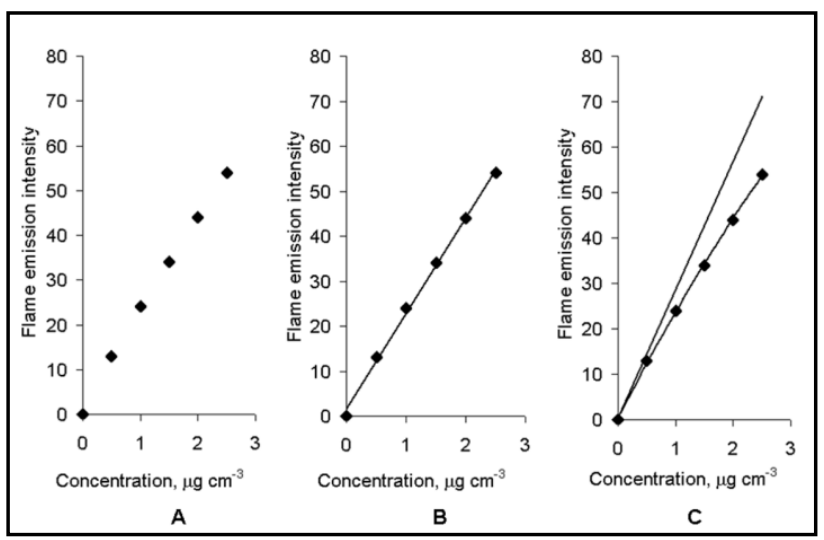

Figure 1. Graph curvature of flame emission intensity function vs. concentration is a symptom for availability of self-absorption. A - experimental points. $\mathrm{B}-$ linear interpretation of the experimental points. $\mathrm{C}$ nonlinear interpretation of the experimental points. Straight line on Fig.1C represents a graph of exited atoms emission intensity as a function of concentration

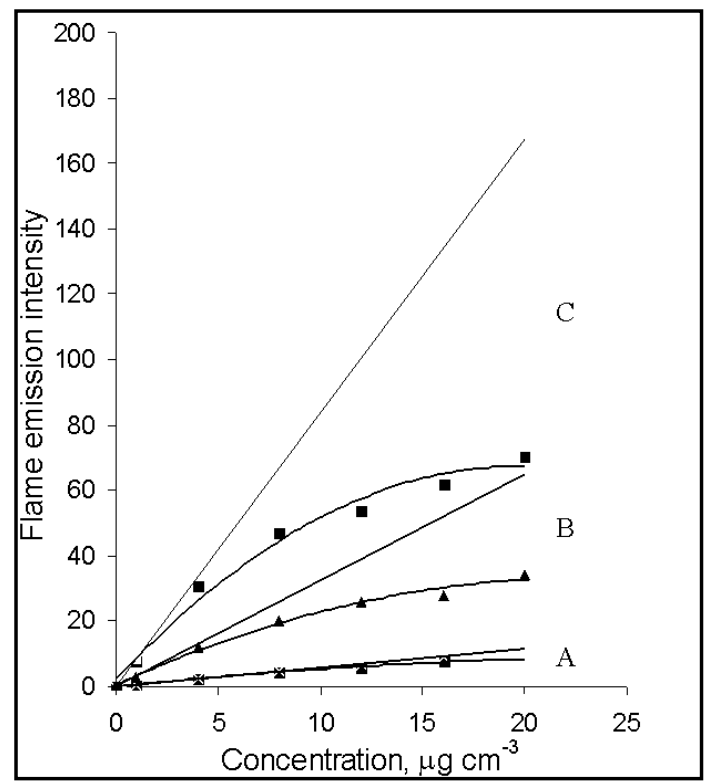

Figure 2. Dependency of flame emission intensity on concentration and flame dimensions. Straight lines are in absence of self-absorption and curved lines are in presence of self-absorption. Graphs are achieved with burners having burner heads with different total opening section area as follows: A - $14.7 \mathrm{~mm}^{2}, \mathrm{~B}-45.5 \mathrm{~mm}^{2}, \mathrm{C}-120.7 \mathrm{~mm}^{2}$

These data is usually evaluated using a linear function as shown in Fig. 1 B. Among the data points there is some variation from a straight line (correlation coefficient $\mathrm{R}^{2}=$ 0.9969). Nevertheless these data are achieved for experi- 
mental conditions, that are appropriate for flame emission process but not for self-absorption process, the influence of self-absorption cannot be excluded. The same experimental data are better described (correlation coefficient $\mathrm{R}^{2}=$ 0.9986 ) by a nonlinear function, see Fig.1C. This confirms availability of the self-absorption process. Because of the self-adsorption process, the intensity of the light that leaves the flame $I_{f}$ has lower values than those of the initial emission intensity $I_{a t}$ of the exited atoms. The straight line presented in Fig. 1C is intensity $I_{a t}$ as a function of concentration. To the extent that self-absorption process occurs, the difference between intensity $I_{a t}$ of the exited atoms and flame intensity $I_{f}$ increases. Therefore the self-absorption process can be easy evaluated as the difference between these two intensities, respectively by their functions of analyte concentration.

As it can be seen in the Fig 2, self-adsorption increases at higher concentrations and is dependent on the kind of burner head used to make the flame.

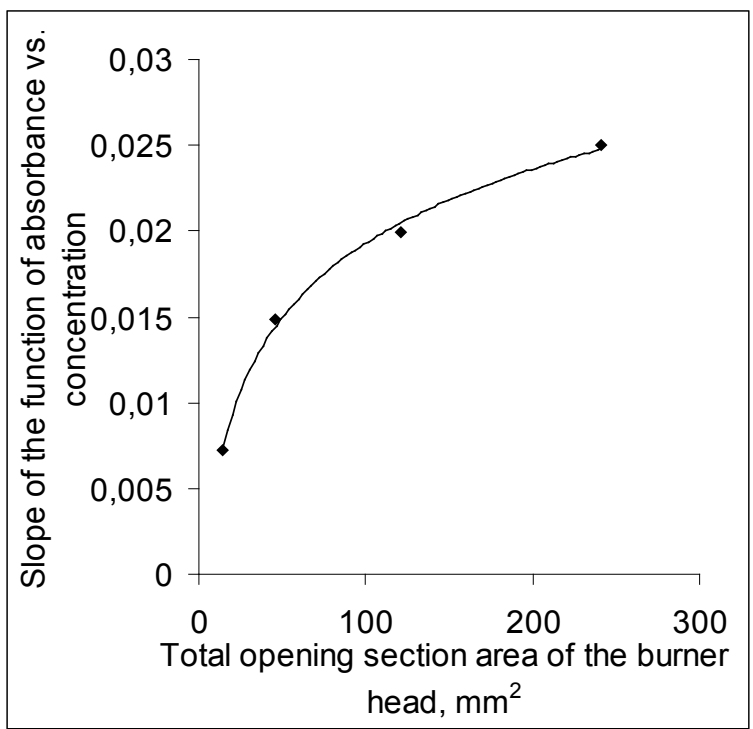

Figure 3. Sensitivity of flame atomic absorption determination as a function of total opening section area of the burner head

Dependence of absorptivity value on burner head design was investigated in order to determine the most suitable burner head for flame atomic absorption analysis. Total opening area of the burner head appears to be a proper parameter for this purpose. Fig.3 presents sensitivity of analy$\mathrm{sis}$ (slope of the function of absorbance vs. concentration) as a function of total opening section area of the burner head used. The comparis on of the second graph point (that corresponding to an opening area of $45.5 \mathrm{~mm}^{2}$ ) with the first point (corresponding to an opening area of $14.7 \mathrm{~mm}^{2}$ ) shows 0.65 units increase in sensitivity per unit opening area, while for the third point, with an opening area of 120.7 $\mathrm{mm}^{2}$, compared with the second one, the increase is 0.51 sensitivity units per unit opening area. For the fourth point, compared to the third one, the increase in sensitivity is only 0.042 sensitivity units per unit opening area. That means the sensitivity increases when opening area increases but this increase is less for bigger section area. In principle the proper burner head has to poss ess an opening area that corresponds to maximal sensitivity, but the increase in opening area is justified if it leads to significant increase in sensitivity. The burner head $2 \mathrm{MBH} 120.7$ is used for further experiments in the work.

The emission burner head MBH 120.7 and absorption burner head 3SBH 120 have approximately one and the same total opening area, respectively $120.7 \mathrm{~mm}^{2}$ and 120 $\mathrm{mm}^{2}$, and therefore approximately one and the same sensitivity (slope values of 0.0199 and 0.0211 respectively). That shows slight influence of flame shape over sensitivity.

Sensitivity not only depends on burner head dimensions, and thus on flame dimensions, but on the part of the flame light that reaches the monochromator. Sensitivity of analysis as a function of slit width of the spectrometer is illustrated in Fig 4. The greatest possible slit width of the spectrometer has to be recommended if that does not cause any spectral influence.

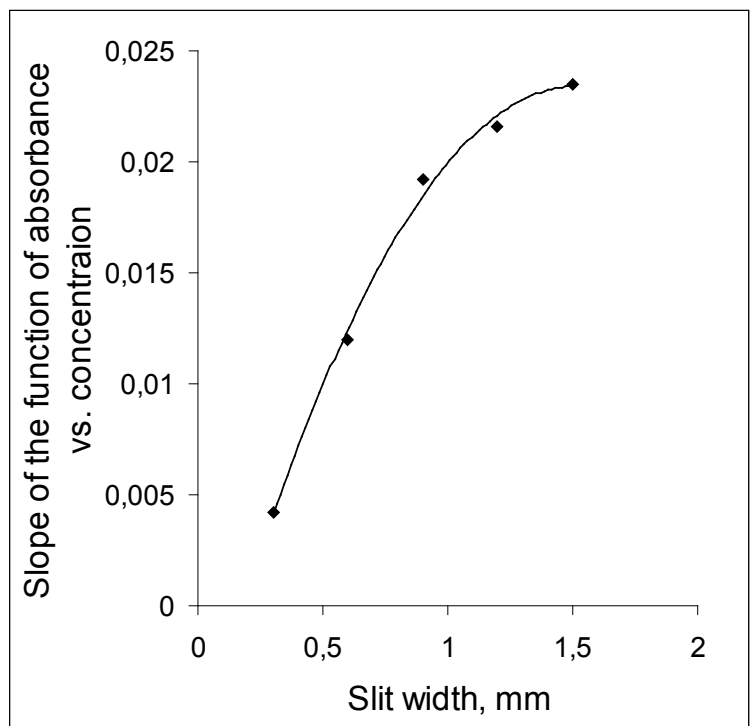

Figure 4. Sensitivity of flame atomic absorption determination as a function of slit width of the spectrometer

The investigations described above are performed with burner heads, each one designed for different work conditions (gas mixture composition, fuel and oxidant gas flow rates). One and the same fuel gas and oxidant gas in the gas mixture have to be used in order for the investigations with different burner heads, as described above, to be done correctly. For safety purposes, liqu id flame fuel is used instead of gas fuel. We have adequate experience for working with liquid fuel[6]. The use of liquid fuel eliminates the hazard of creating explosive gas mixtures. Ethyl alcohol is used as a liquid flame fuel and is applied as an alcohol vapor / a ir flame. This flame is similar to a propane / air flame but its flame temperature is probably lower than the propane / air flame temperature. For this reason determinations were done with only certain alkali and alkaline earth elements. Specifically, lithium, potassium, sodium, calcium, barium are analyzed. 
Table 1 presents data from the atomic absorption analysis of these elements, by the method presented here, using burner head 2MBH 120.7 and alcohol vapor/a ir flame. The results for characteristic concentration are not good enough. Usage of an old model spectrometer consisting of parts of other outdated spectrometers, having not quite suitable burner and an attached vaporizer, detrimentally affected the results for absorbance determination.

Table 1. Data for Flame Atomic Absorption Analysis based on self-absorption and using the flame as a light emission source

\begin{tabular}{|c|c|c|c|}
\hline $\begin{array}{c}\text { Element } \\
\text { to be } \\
\text { analyzed }\end{array}$ & $\begin{array}{c}\text { Absorbance vs. } \\
\text { concentration } \\
\text { equation }\end{array}$ & $\begin{array}{c}\text { Correlation } \\
\text { coefficient } \\
\mathrm{R}^{2}\end{array}$ & $\begin{array}{c}\text { Characterist ic } \\
\text { concentration } \\
\mu \mathrm{g} \mathrm{cm}^{-3}\end{array}$ \\
\hline Lithium & $\begin{array}{c}A=4.38 \times 10^{-3} C- \\
5 \times 10^{-5}\end{array}$ & 0.9985 & 1.00 \\
\hline Potassium & $\begin{array}{c}A=9.42 \times 10^{-3} C- \\
1.8 \times 10^{-3}\end{array}$ & 0.9938 & 0.47 \\
\hline Sodium & $\begin{array}{c}A=11.6 \times 10^{-3} C- \\
2 \times 10^{-5}\end{array}$ & 0.9974 & 0.38 \\
\hline Calcium & $\begin{array}{c}A=3.85 \times 10^{-4} C+ \\
2 \times 10^{-4}\end{array}$ & 0.9911 & 11.4 \\
\hline Barium & $A=9.69 \times 10^{-5} C-$ & 0.9915 & 45.5 \\
\hline
\end{tabular}

Another reason for the poor sensitivity achieved is the low temperature of the alcohol / air flame used. The method presented here is an absorption method but is based on a measurement of a flame emission signal, so this method has to be performed at experimental conditions recommended in literature for performing flame emission spectrometry but not for flame absorption spectrometry. The flame temperature has to be high enough to ensure not only atomization but also to achieve excitation of the atoms. Acetylene / nitrous oxide flame satisfies these requirements for almost all elements to be analyzed.

Using sodium analysis, the influence of the flame temperature on the sensitivity of analysis is studied. Two different flames having different flame temperature, alcohol / air flame and acetylene / a ir flame, are applied. One and the same burner head, MBH14.7, is used for this test. Characteristic concentration achieved with alcohol / air flame is $0.61 \mu \mathrm{g} \mathrm{cm}^{-3}$, while that for acetylene / air flame is $0.19 \mu \mathrm{g}$ $\mathrm{cm}^{-3}$, that is about three times lower.

In principal analytical characteristics for atomic absorption analysis, such as sensitivity and detection limit, for the method presented here, must be of the same range as for flame emission spectrometry. The reason is that the flame emission signal is measured while absorption is only calculated.

Analysis of sodium is a good example for comparing sensitivity of the method proposed here and ordinary flame atomic absorption, because the acetylene / air flame is recommended for both methods. Sensitivity of the ordinary method is better (characteristic concentration is $0.038 \mu \mathrm{g}$ $\mathrm{cm}^{-3}$ ) than the proposed method (characteristic concentration is $0.059 \mu \mathrm{g} \mathrm{cm}^{-3}$ ). A single slot burner head, used for this test, is not suitable for presented here method because of the low total opening area of the burner head (the influ- ence of the burner head upon the sensitivity and respectively over the characteristic concentration is discussed in details in the paper). Further improvement in the sensitivity of analysis can be achieved if a special burner head is constructed.

The advantages of the method presented here are its simplicity and lower equipment costs. The method is simply performed because only one measurement is made (that of a flame emission intensity) while for common flame absorption analysis two measurements of intensities have to be done. There is no need of a separate light source and all systems connected with it. In fact, flame atomic absorption analysis can be performed with a flame emission spectrometer.

Presented method of atomic absorption analysis is based on measuring atomic emission intensity signal and using self-absorption process to determine absorption. This method in principal could be applied for atomic emission methods for which self-absorption is taken place. It is known that in ICP (a modern atomic emission method) self-absorption exists[7], so the proposed method might be useful in association with ICP method.

\section{REFERENCES}

[1] Colin A. Watson, Use of Improved Design and Boosted-discharge Hollow-cathode Lamps, Royal Society of Chemistry Publishing, Journal of Analytical Atomic Spectrometry, vol.5, no.3, pp.404-414, 1988.

[2] David Butcher, Lasers as Light Sources for Analytical Atomic Spectrometry, Taylor and Francis Ltd, Applied Spectroscopy, Reviews, vol. 42, no.6, pp. 543-562, 2007.

[3] H. Becker-Ross, S. Florek, U. Heitmann, M.D. Huang, M. Okruss, B. Radziuk, Continuum Source Atomic Absorption Spectrometry and Detector Technology: A Historical Perspective, Elsevier, Spectrochimica Acta, Part B, Review, vol.61, pp. 1015-1030, 2006.

[4] Clifton P Calloway Jr, Bradley T Jones, Atomic Absorption Spectrometry With a Flame Emission Source, Pergamon, Spectrochimica Acta, Part B, Review, vol. 49B, nos 12-14, pp. 1707-1715, 1994.

[5] Anastas D. Dakashev, A Method for Performing Atomic Absorption Analysis Using the Atomizer as a Light Emission Source, Bulgarian Patent No 110535, 27.11.2009.

[6] Anastas D.Dakashev, Maria M. Nedelcheva, Krasimira A. Stancheva, Flame Emission Spectrometric Analysis Using Ethil Alchohol as a Flame Fuel, University Publishing, Annual Assen Zlatarov University, vol. 37, no.1, pp.15-18, 2008.

[7] Matthieu Chausseau, Emmanuelle Poussel, J.M. Mermet, Self-absorption effects in radially and axially viewed inductively coupled plasma-atomic emission spectrometry - the key role of the operating conditions, Springer, Fresenius' Journal of Analy tical Chemistry, vol.370, no. 4, pp. 341-347, 2001. 